\title{
2-Aminoacetophenone as a potential breath biomarker for Pseudomonas aeruginosa in the cystic fibrosis lung
}

\author{
Amy J Scott-Thomas ${ }^{1 *}$, Mona Syhre ${ }^{2}$, Philip K Pattemore ${ }^{1}$, Michael Epton ${ }^{3}$, Richard Laing ${ }^{3}$, John Pearson ${ }^{1}$, \\ Stephen T Chambers ${ }^{3}$
}

\begin{abstract}
Background: Pseudomonas aeruginosa infections are associated with progressive life threatening decline of lung function in cystic fibrosis sufferers. Growth of Ps. aeruginosa releases a "grape-like" odour that has been identified as the microbial volatile organic compound 2-aminoacetophenone (2-AA).

Methods: We investigated 2-AA for its specificity to Ps. aeruginosa and its suitability as a potential breath biomarker of colonisation or infection by Solid Phase Micro Extraction and Gas Chromatography-Mass Spectrometry (GC/MS).

Results: Cultures of 20 clinical strains of Ps. aeruginosa but not other respiratory pathogens had high concentrations of 2-AA in the head space of in vitro cultures when analysed by GC/MS. 2-AA was stable for 6 hours in deactivated glass sampling bulbs but was not stable in Tedlar ${ }^{\circledR}$ bags. Optimisation of GC/MS allowed detection levels of 2-AA to low pico $\mathrm{mol} / \mathrm{mol}$ range in breath. The 2-AA was detected in a significantly higher proportion of subjects colonised with Ps. aeruginosa 15/16 (93.7\%) than both the healthy controls 5/17 $(29 \%)(p<0.0002)$ and CF patients not colonised with Ps. aeruginosa 4/13(30.7\%) $(\mathrm{p}<0.001)$. The sensitivity and specificity of the 2-AA breath test compared to isolation of Ps. aeruginosa in sputum and/or BALF was $93.8 \%(95 \% \mathrm{Cl}, 67-99)$ and $69.2 \%(95 \% \mathrm{Cl}, 38-89)$ respectively. The peak integration values for 2-AA analysis in the breath samples were significantly higher in Ps. aeruginosa colonised subjects (median 242, range 0-1243) than the healthy controls (median 0, range 0-161; $\mathrm{p}<0.001$ ) and CF subjects not colonised with Ps. aeruginosa (median 0, range 0-287; $<<0.003$ )

Conclusions: Our results report 2-AA as a promising breath biomarker for the detection of Ps. aeruginosa infections in the cystic fibrosis lung.
\end{abstract}

\section{Background}

Pseudomonas aeruginosa is a Gram negative bacterium that produces a sweet "grape-like" odour during growth. In 1966 Mann [1] identified this compound as 2 -aminoacetophenone (2-AA) by thin-layer chromatography. Cox \& Parker [2] confirmed 2-AA as the compound responsible for this odour and we have also successfully detected and identified 2-AA in the headspace of in vitro cultures using gas chromatography/

\footnotetext{
* Correspondence: amy.scott-thomas@otago.ac.nz

${ }^{1}$ University of Otago, Christchurch School of Medicine and Health Sciences, Department of Pathology, P.O. Box 4345, Christchurch, 8140, New Zealand
} Full list of author information is available at the end of the article mass spectrometry (GC/MS) (Figure 1). 2-Aminoacetophenone is a small, volatile aromatic compound with a molecular weight of $135 \mathrm{~g} / \mathrm{mol}$ (Figure 1). It is an intermediate product in the biosynthesis of quinazolines, which branches from the tryptophan catabolic pathway [3]. 2-Aminoacetophenone is consistently produced by multiple Ps. aeruginosa strains, on all culture media, and is a major metabolite [2-4], but the biological significance of this compound is unknown [2]. There is a single report of 2-AA detected in the headspace of Escherichia coli cultures [5] but 2-AA is not known to be produced by other respiratory pathogens. Because of the well described evidence of the production of 2-AA by Ps. aeruginosa and its apparent

\section{() Biomed Central}




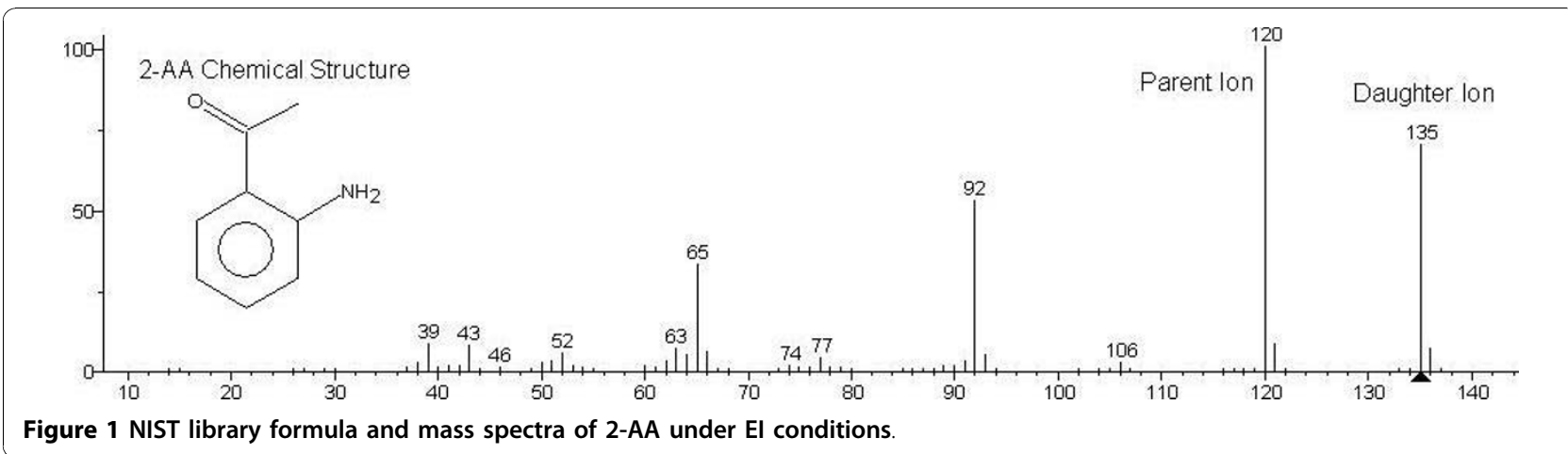

specificity we thought it may be useful as a volatile biomarker for infection and/or colonisation of the lung.

Cystic Fibrosis (CF) is an autosomal recessive disease affecting 1 in 3300 live Caucasian births [6]. The secretion of hyperviscous mucus in the CF-affected lung provides $P$ s. aeruginosa with a nutritionally rich growth environment, where it often grows to high cell densities $\left(>10^{9}\right.$ cells $/ \mathrm{ml}$ sputum) $[7,8]$. Although many other bacterial species persist and grow in the CF lung, chronic Ps. aeruginosa infection correlates with declining lung function and high mortality rates $[4,9]$.

None of the current methods for detecting Ps. aeruginosa infections in CF patients are satisfactory. The culture of broncho-alveolar lavage fluid (BALF) is currently the reference standard for lower respiratory tract colonisation and infection [10]. However, this procedure is invasive and requires general anaesthetic in the very young. While BALF is not routinely performed, it has been used successfully in screening studies of both newly diagnosed and very young CF patients $[11,12]$. Cultures of sputum samples are significantly less sensitive than BALF [13] and cultures of the oropharynx have poor sensitivity and do not reliably predict the presence of organisms in the lower airways [14].

We have therefore undertaken an investigation to determine whether 2-AA is consistently produced by Ps. aeruginosa and other respiratory pathogens during in vitro culture and if 2-AA is detectable in the breath of CF patients colonised with Ps. aeruginosa.

\section{Methods \\ Materials}

2-Aminoacetophenone, hexamethyldisilazane and $1 \mathrm{~L}$ glass sampling bulbs were purchased from Sigma Aldrich, St. Louis, Missouri, United States of America. $2 \mathrm{~L}$ Tedlar ${ }^{\circ}$ bags were purchased from SKC Inc., PA, USA. Vials of tobramycin (Hospira, Wellington, New Zealand) and colistin (Link, Australia) for clinical use were obtained from the Christchurch Hospital Pharmacy, New Zealand.

\section{Solid Phase Micro Extraction}

SPME fibres $\left(60 \mu \mathrm{m}\right.$ Carbowax $\left.{ }^{\mathrm{Tx}} / \mathrm{PEG}\right)$ (Supelco, Bellefonte, Pennsylvania, United States of America) were used to pre-concentrate 2-AA from the headspace of cultures and breath samples. Each SPME fibre was preconditioned in a hot injector at $250^{\circ} \mathrm{C}$, a test chromatogram was recorded and the clean and activated fibre was then exposed into the sample headspace.

\section{Detection of 2-AA by GC/MS}

A Saturn 2200 system (Varian, Palo Alto, USA) was used to perform the GC/MS analysis. A Zebron ZBWax $30 \mathrm{~m} \times 0.25 \mathrm{~mm} \times 0.25 \mathrm{~mm}$ (Phenomenex, Auckland, New Zealand) was connected into a Programmable Temperature Vaporiser (PTV-1079) injector. The temperatures of the injector, ion trap, manifold and transfer line were $250,200,60$ and $250^{\circ} \mathrm{C}$, respectively. The oven program commenced at $60^{\circ} \mathrm{C}$ for 2 minutes and was raised to $250^{\circ} \mathrm{C}$ at a rate of $10^{\circ} \mathrm{C} / \mathrm{min}$, at which the temperature was maintained for a further 2 minutes. Helium flow was set at a constant rate of $1.2 \mathrm{ml} / \mathrm{min}$. The split vent was opened to a ratio of 1:50 after $1 \mathrm{~min}$. Initial full scan spectra were obtained with a mass to charge $(\mathrm{m} / \mathrm{z})$ range of 40-650 utilising electron impact (EI) (Figure 2). A change to chemical ionisation (CI) with methanol as the precursor ion in full scan mode increased sensitivity for 2-AA in comparison to EI.

In order to analyse 2-AA in breath it was necessary to utilise the MS-MS capabilities of the ion trap due to matrix and very low detection level requirements. Ion preparation for MS-MS analysis was in CI mode; the selected parent ion was $\mathrm{m} / \mathrm{z} 135$ with an isolation window of $\mathrm{m} / \mathrm{z} 3$; excitation storage level was 65 ; excitation amplitude was 48; the resulting MS-MS spectra featured three main peaks at $\mathrm{m} / \mathrm{z} 118$ (100\%), $\mathrm{m} / \mathrm{z} 136$ (90\%) and $\mathrm{m} / \mathrm{z}$ 94 (45\%) (Figure 3). Further sensitivity was achieved by additional fragmentation using $\mathrm{MS}^{3}$. The selected daughter ion was $\mathrm{m} / \mathrm{z} 118$ with an isolation window of $\mathrm{m} / \mathrm{z} 3$; excitation storage level was 65 ; excitation amplitude was 48; the resulting $\mathrm{MS}^{3}$ spectra featured one main peak at m/z 118 (100\%) (Figure 4). 


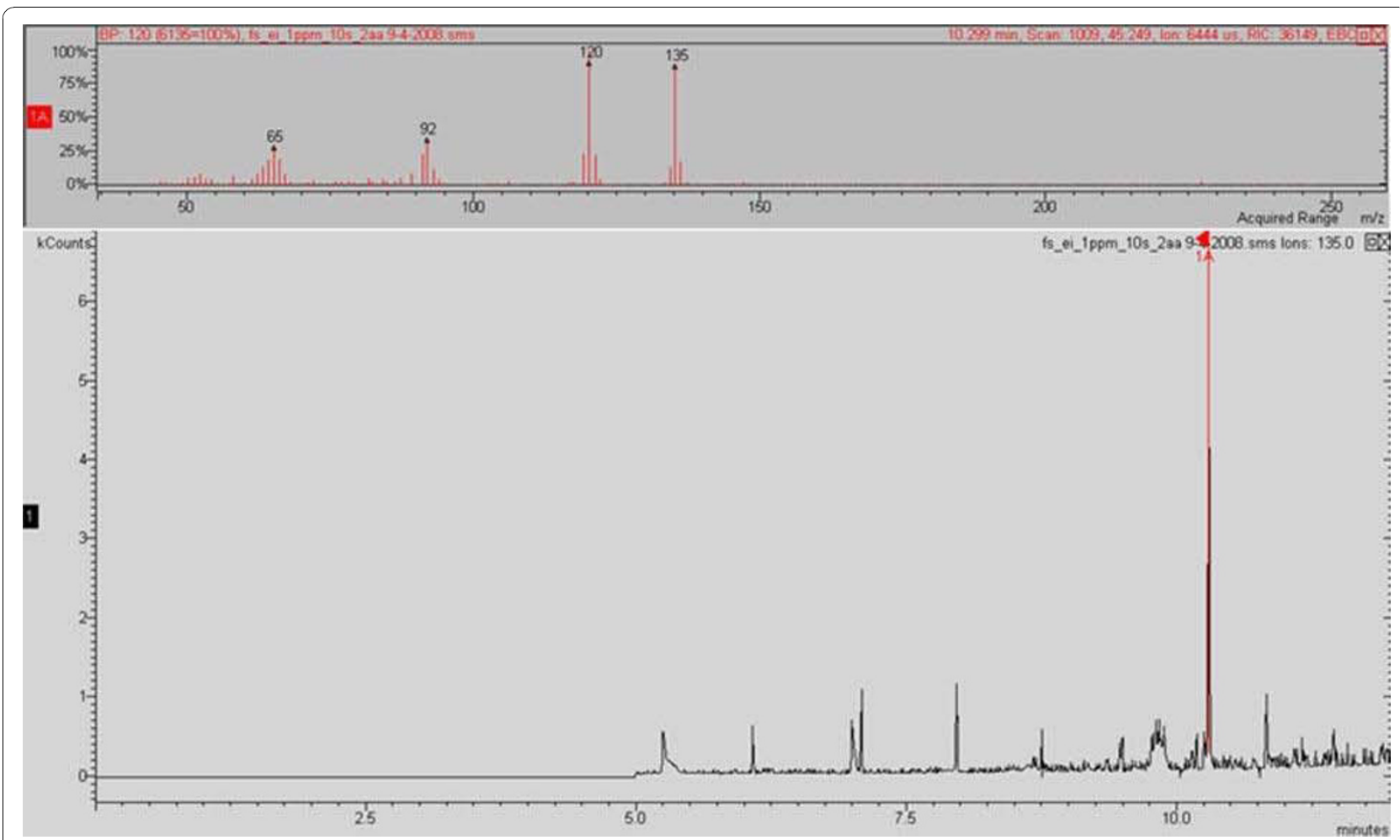

Figure 2 Experimental spectra for 2-AA, obtained under GC/MS El conditions, from in vitro Ps. aeruginosa cultures.

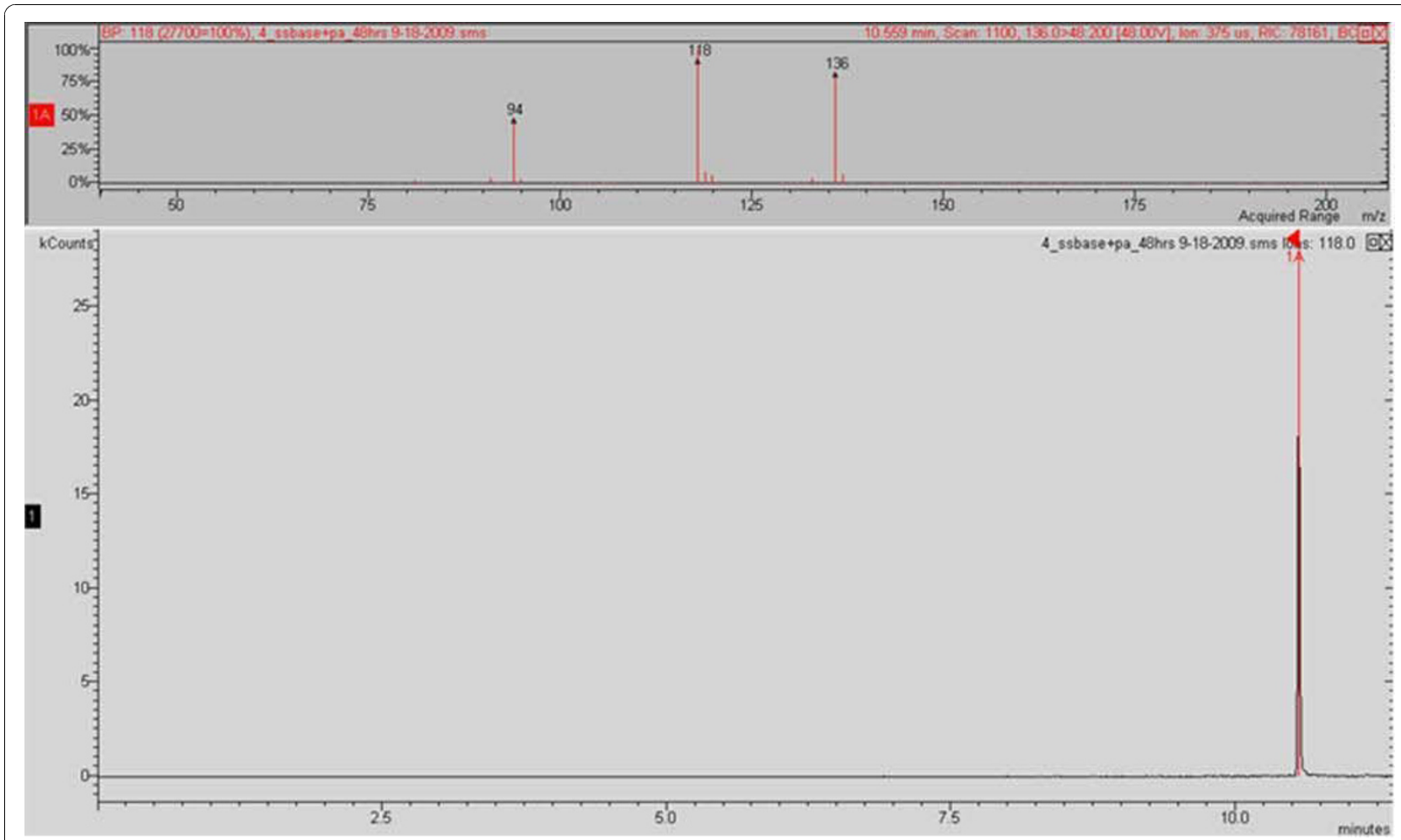

Figure 3 Experimental spectra for 2-AA, obtained under GC/MS-MS $\mathrm{Cl}$ conditions, from in vitro Ps. aeruginosa cultures. 


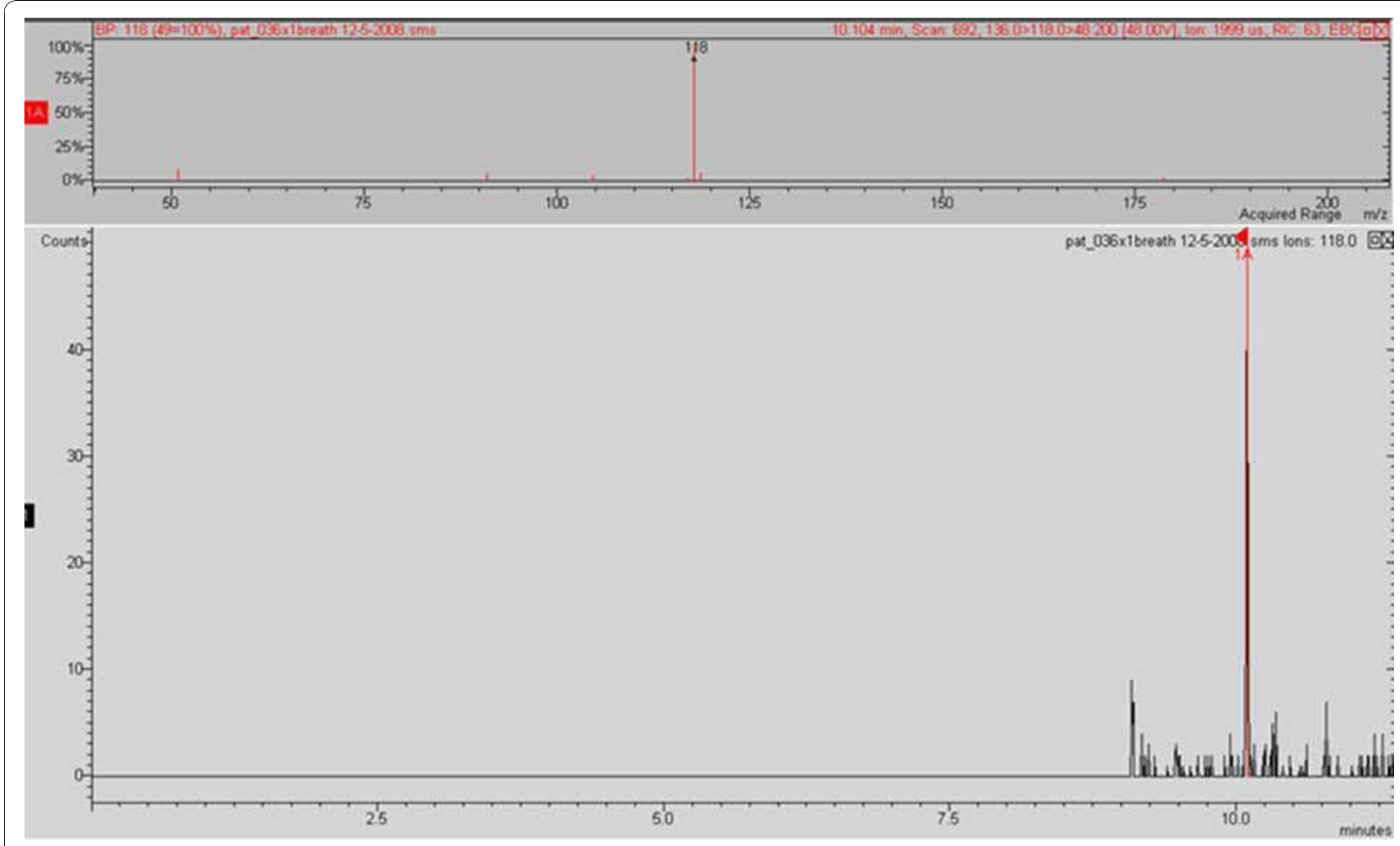

Figure 4 Experimental spectra for 2-AA, obtained under GC/MS3 $\mathrm{Cl}$ conditions, from a breath samples positive for 2-AA.

\section{Micro-organisms}

The following species were tested; Ps. aeruginosa (20 strains), E. coli (3 strains), Burkholderia multivorans (3 strains), Haemophilus influenzae (3 strains), Legionella pneumophila (3 strains), Moraxella catarrhalis (3 strains), Pseudomonas fluorescens (3 strains), Staphylococcus aureus (3 strains), Streptococcus pneumoniae (3 strains) and Aspergillus fumigatus (3 strains). All strains were isolated and identified from a variety of respiratory specimens by Canterbury Health Laboratories, Christchurch, New Zealand by standard techniques [15]. B. multivorans strains were identified by API $20 \mathrm{NE}$ and 16S rRNA sequencing [16]. All strains were stored at $4^{\circ} \mathrm{C}$ and re-plated regularly.

\section{Culture methods}

All micro-organisms were cultured in $50 \mathrm{ml} \mathrm{screw}$ top bottles (Alltech Associates Inc. Illinois, United States of America) containing $10 \mathrm{ml}$ of medium. Columbia Sheep Blood agar, Sheep Blood agar, and Mueller-Hinton agar were purchased from Fort Richards Laboratories Ltd, Auckland, New Zealand. Luria Bertani agar and M9 Minimal Media broth was prepared in the laboratory as specified in the Handbook of Microbiological Media [17]. The open screw cap contained a Teflon lined silicone septa. Two replicates of each micro-organism strain were inoculated into the media separately
(0.5 MacFarlane Standard). The bottles were sealed tightly and incubated for at least 24 hours at $37^{\circ} \mathrm{C}$, aerobically. The headspace gas was sampled using SPME by penetrating the septum.

\section{Drug testing for 2-AA}

The agents were reconstituted into a glass vial with a Teflon septum according to the manufacturer's recommendations. The SPME fibre was introduced into the headspace through the septum for 5 minutes and then desorbed into the GC/MS instrument for analysis by full scan and MS-MS.

\section{Environmental air sampling}

Levels of environmental 2-AA were determined from two sites where breath samples were collected (Respiratory Ward and Out Patients at Christchurch Hospital, New Zealand). Ten clean glass bulbs were prepared for environmental air sampling with a vacuum of $550 \mathrm{~mm}$ $\mathrm{Hg}$. Bulbs were transported to sampling sites and the stop cocks opened releasing the vacuum and in turn sampling the environmental air. As soon as the sample was obtained the stopcocks were closed and the bulbs transported back to the laboratory for analysis. SPME fibres were inserted via the septa within 30 minutes. All sampling devices were incubated at $37^{\circ} \mathrm{C}$ for 24 hours before analysis. 


\section{Subjects}

Written informed consent was obtained from all subjects. Control subjects were healthy, non-smoking adults who had no known respiratory disease, and were not taking prescription or over the counter medication. Subjects (both adults and children) with clinically stable CF were identified by staff of specialist respiratory services at Christchurch Hospital, New Zealand. All subjects had CF diagnosed either by genetic analysis or at least three positive sweat tests $(>60 \mathrm{mmol} / \mathrm{L})$ and progressive obstructive airway disease characterised by a persistent, productive cough, hyperinflation of the lung fields on chest radiograph, and obstructive pulmonary function tests. Computerised medical records were reviewed for the past medical history and laboratory investigations and the diagnoses were confirmed by a specialist respiratory physician. CF patients with Ps. aeruginosa had to have at least two positive sputum and/or BALF tests for Ps. aeruginosa for inclusion into this group. The micro-organisms present in the respiratory secretions of CF patients were identified by culture at Canterbury Health Laboratories, Christchurch, New Zealand. A drug and dietary history was taken using a standardised questionnaire and entered into a data sheet.

\section{Breath sampling}

Sampling was done between $8.30-9.30$ am or 1.30$3.30 \mathrm{pm}$ and the subjects food and/or fluid ingestion, over the 12 hours prior to breath collection, was recorded. Breath samples were obtained more than two hours after consumption of any foods.

All healthy controls and subjects with CF were studied in facilities sourced from a common air conditioning system. No other filters or control systems were used. Breath samples were collected by forced expiration of five breaths into the $1 \mathrm{~L}$ silanized glass sampling bulb without a nose clip or saliva trap. Stop cocks at each end were opened while breath was collected by forced expiration directly into the bulb without any additional mouthpiece. Some rest was allowed between breaths but all breath samples were collected within two minutes. As soon as the breath samples were collected the stop cocks were closed and the device was ready for analysis. Collection bulbs were taken to the GC/MS laboratory within 30 minutes of collection, SPME fibres were inserted via the septa within two hours. All sampling devices were incubated at $37^{\circ} \mathrm{C}$ for 24 hours before analysis.

\section{Statistical analysis}

Degradation of 2-AA in the glass bulb was tested with the I-test adapted for unequal variances. A five parameter log logistic (5PL) model was fitted to the data and the $\mathrm{EC}_{50}$ estimated using the $\mathrm{R}$ package drc [18]. The 5PL model may be parameterised by equation 1 where $c$ and $d$ are the minimum and maximum concentrations respectively, $d$ the hill slope, $e$ the $\mathrm{EC}_{50}$ and $f$ the asymmetry parameter.

$$
\text { Concentration }=c+\frac{d-c}{\left[1+\left(\frac{\text { time }}{e}\right)^{b}\right]^{f}}
$$

The four parameter log logistic (4PL) model has the same parameterisation as the $5 \mathrm{PL}$ with $f$ set to 1 , forcing symmetry about the point of inflection. ANOVA shows that the 5PL had significantly better fit to the data than the 4PL model $(\mathrm{p}<0.0002)$. The estimated minimum concentration for the 5PL model was -3.18 (SE 8.99) hence we fitted a 5PL model with the minimum concentration, $c$, constrained to 0 to aid interpretability. Table 1 shows the Akaike Information Criterion (AIC) for each model and the estimated $\mathrm{EC}_{\mathrm{p}}$ where the $95 \%$ confidence intervals were estimated by the delta method [18]. The AIC is a measure of fit which penalises additional parameters; lower values indicate better fit so the table shows that the 5PL model fits better than the 4PL while fixing the minimum concentration provides a marginal improvement. The colonised subjects were compared with both control groups for presence versus absence (below threshold of 2-AA detection) in breath with Fisher's exact test. The Mann-Whitney test was used to compare the peak integration values of the three group's breath tested. Statistical tests and modelling was performed in $\mathrm{R}$ version 2.10 [19].

\section{Ethical approval}

Ethical approval for the study was obtained from the Upper South Island Ethics Committee, New Zealand and the study was conducted in accordance with the standards for clinical research of the University of Otago, New Zealand.

\section{Results}

\section{2-AA production by bacteria in vitro}

Twenty clinical isolates of Ps. aeruginosa (including 12 mucoid strains) obtained from a variety of respiratory samples submitted to Canterbury Health Laboratories were tested. 2-Aminoacetophenone was detected in the

Table 1 2-AA degradation models

\begin{tabular}{lrccc}
\hline Model & AIC & EC $_{\mathbf{5 0}}$ & $\mathbf{9 5 \%} \mathbf{C l}$ & \\
\hline $5 P L C=0$ & 144.84 & 11.73 & 10.94 & 12.52 \\
$5 P L$ & 146.48 & 12.38 & 9.03 & 15.73 \\
$4 P L$ & 162.30 & 9.71 & 8.92 & 10.49 \\
\hline
\end{tabular}


head space in "high" quantities (in the milli $\mathrm{mol} / \mathrm{mol}$ range) in all isolates after 24 hours incubation on Sheep Blood agar. 2-Aminoacetophenone was detected 10 out of 10 times from the head space of a single Ps. aeruginosa strain cultured on Columbia Sheep Blood agar. This same strain also produced 2-AA when cultured on Mueller-Hinton agar, Luria Bertani agar, M9 Minimal Media broth and laboratory tap water. 2-Aminoacetophenone was not detected from the head space of $H$. influenzae, L. pneumophila, M. multivorans, Ps. fluorescens, B. multivorans, S. pneumoniae and A. fumigatus strains upon culturing on Columbia Sheep Blood agar, Sheep Blood agar and Mueller-Hinton agar. S. aureus and E. coli strains did not produce 2-AA when grown on Sheep Blood agar or Mueller-Hinton agar. However, one out of three $S$. aureus strains and two out of three E. coli strains produced trace levels of 2-AA (low pico $\mathrm{mol} / \mathrm{mol}$ ) on Columbia Sheep Blood agar.

\section{2-AA stability in breath collection systems}

$2 \mathrm{~L}$ Tedlar ${ }^{\circ}$ bags and $1 \mathrm{~L}$ deactivated glass bulbs were spiked $(10 \mu \mathrm{mol})$ with 2 -AA reference standard and were analysed for stability of 2 -AA at $37^{\circ} \mathrm{C}$. Within two hours of spiking, only $10 \%$ of the initial concentration was detectable in the Tedlar bags and no 2-AA was detectable after 24 hours. 2-Aminoacetophenone was found to be stable over two hours in the glass sampling bulb and after 24 hours approximately $40 \%$ was still present. The preliminary comparison showed the superiority of the glass sampling bulbs for 2-AA. To determine the "half-life" of 2-AA in the glass sampling bulb system, 2-AA was spiked into the glass bulbs (three replicates) at a concentration of $10 \mu \mathrm{mol}$. The resulting time degradation is illustrated in Figure 5. The $\mathrm{EC}_{50}$ for 2-AA was

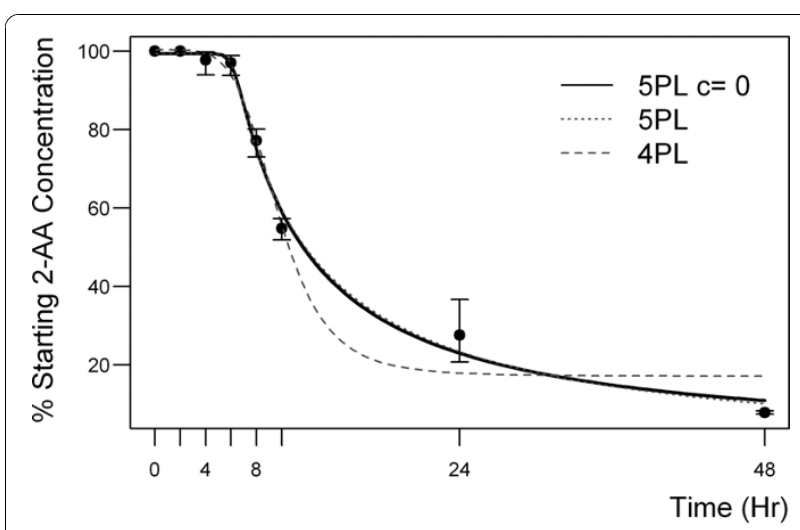

Figure 5 A time degradation graph of 2-AA in glass sampling bulbs. The 2-AA levels (mean and SD) from three replicate experiments are shown and a five parameter logistic function fitted to the degradation curve $(11.73$ hours $(95 \% \mathrm{Cl} 10.94,12.52)$. 2-AA levels at 8 and 10 hours were significantly less $(P<0.001)$ than four and six hours. estimated to be 11.73 hours (95\% CI 10.94, 12.52). A peak integration area of 88 was determined as the lower limit of detection (detection limit) for 2-AA in the $1 \mathrm{~L}$ glass sampling bulb filled with ambient air. This peak integration value was determined to be a concentration $50 \mathrm{pmol} / \mathrm{mol}(\mathrm{n}=15$ with a standard deviation of $4.8 \mathrm{pmol} / \mathrm{mol})$.

\section{Environmental air sampling}

Air samples were analysed from the two collection sites in $\mathrm{MS}^{3}$ mode to ensure no environmental contamination of breath samples with 2-AA. The levels of 2-AA detected in the environmental samples were well below the threshold of detection in all samples analysed from both sites.

\section{Study population}

The clinical characteristics, medication use and microorganisms isolated from CF sputum samples are shown in Tables 2, 3 and 4. As expected, subjects colonised with Ps. aeruginosa were associated with older age, more complications of CF with more anti-pseudomonal and antimicrobial therapy. S. aureus colonisation was more frequent in those who were not colonised with $P$ s. aeruginosa $11 / 13$ (85\%) compared to those that were colonised with Ps. aeruginosa $7 / 16$ (43\%). Food and fluid intake in the previous 12 hours was similar in both the subject and the control group (Table 5).

\section{Detection of 2-AA in breath samples}

The 2-AA was detected in a significantly higher proportion of subjects colonised with Ps. aeruginosa 15/16 (93.7\%) than both the healthy controls 5/17 (29\%) (p < 0.0002) and CF patients not colonised with Ps. aeruginosa $4 / 13(30.7 \%)(\mathrm{p}<0.001)$. The sensitivity and specificity of the 2-AA breath test compared to isolation of Ps. aeruginosa in sputum and/or BALF was $93.8 \%$ (95\% CI, 67-99) and $69.2 \%$ (95\% CI, 38-89) respectively. The peak integration values for 2-AA analysis in the breath samples are shown in Figure 6. These values were significantly higher in the breath of patients colonised with $P$ s. aeruginosa (median 242, range 0-1243) than the healthy controls (median 0 , range $0-161 ; \mathrm{p}<0.001$ ) and CF subjects not colonised with Ps. aeruginosa (median 0, range 0 -287; $\mathrm{p}<0.003$ ).

\section{Discussion}

There is well-established and growing interest in the detection and identification of micro-organisms by measuring their release of volatile organic compounds (VOCs) [20]. Because 2-AA is associated with the "grape-like" smell of Ps. aeruginosa and is more consistently produced than the common pyocyanin [21], it was hypothesized that 2-AA could be a potential breath 
Table 2 Characteristics of subjects included in breath sampling

\begin{tabular}{|c|c|c|c|}
\hline & Healthy controls & CF - Ps. aeruginosa colonised & CF - Ps. aeruginosa non-colonised \\
\hline Number (n) & 17 & 16 & 13 \\
\hline Age years (mean range) & $38(25-54)$ & $26.9(13-59)$ & $14.9(6-43)$ \\
\hline Male (\%) & $38 \%$ & $63 \%$ & $53 \%$ \\
\hline BMI $\left(\mathrm{kg} / \mathrm{m}^{2}\right)$ (mean range) & & $19.07(17.1-24.4)$ & $19.9(14.8-37.2)$ \\
\hline Respiratory function: $\mathrm{FEV}_{1} \%$ & & $44.92(13-93)$ & $77.15(48-99)$ \\
\hline FVC\% & & $59.51(1.14-102)$ & $91.1(55-120)$ \\
\hline \multirow[t]{8}{*}{ Genetic analysis } & & Sweat test (3) & Sweat test (3) \\
\hline & & $\Delta \mathrm{F} 508 / \Delta \mathrm{F} 508(9)$ & $\Delta \mathrm{F} 508 / \Delta \mathrm{F} 508(5)$ \\
\hline & & $\Delta F 508 / G 542 X(3)$ & $\Delta \mathrm{F} 508 / \mathrm{G} 542 \mathrm{X}(1)$ \\
\hline & & $\Delta \mathrm{F} 508 / \mathrm{c} .1339$ delA (1) & C.F508/c.1766+1g (1) \\
\hline & & & $\Delta \mathrm{F} 508 / 3849+10 \mathrm{kbct}(1)$ \\
\hline & & & c.1624g > t(p.g542x)/c.1 \\
\hline & & & $624 g>t(p . g 542 x)(1)$ \\
\hline & & & $\Delta \mathrm{F} 508 / \mathrm{P} 67 \mathrm{~L}(1)$ \\
\hline \multicolumn{4}{|l|}{ Diagnoses } \\
\hline Pancreatic insufficiency & & 15 & 12 \\
\hline Chronic rhinosinuitis & & 9 & 0 \\
\hline Osteopenia & & 8 & 0 \\
\hline Gastro-oesophageal disease & & 7 & 0 \\
\hline CF related diabetes & & 4 & 3 \\
\hline Vitamin A, D \& E deficiency & & 5 & 1 \\
\hline Asthma & & 5 & 0 \\
\hline $\begin{array}{l}\text { Allergic bronchopulmonary } \\
\text { aspergillosis }\end{array}$ & & 0 & 3 \\
\hline $\begin{array}{l}\text { Distal intestinal obstruction } \\
\text { syndrome }\end{array}$ & & 1 & 2 \\
\hline Previous tuberculosis & & 1 & 0 \\
\hline Pancreatitis & & 1 & 0 \\
\hline Chronic nasal polyps & & 0 & 1 \\
\hline Haemoptysis & & 0 & 0 \\
\hline Chronic eczema \& ichthyosis & & 1 & 1 \\
\hline
\end{tabular}

${ }^{\wedge}$ Some CF subjects had multiple diagnoses

Table 3 Medication prescribed to cystic fibrosis patients at time of breath sampling*

\begin{tabular}{lll}
\hline Medication & CF - Ps. aeruginosa colonised $(\mathbf{n}=\mathbf{1 6})$ & CF - Ps. aeruginosa non-colonised $(\mathbf{n}=\mathbf{1 3})$ \\
\hline Creon ${ }^{\oplus}$ & 8 & 13 \\
Tobramycin nebulised & 9 & 1 \\
Colistin nebulised & 7 & 1 \\
Fluticasone propionate & 3 & 5 \\
Salbutamol & 4 & 4 \\
Budesonide-formoterol & 4 & 3 \\
Itraconazole & 2 & 5 \\
Dornase alfa & 3 & 1 \\
Budesonide & 3 & 0 \\
Ciprofloxacin & 0 & 3 \\
Salmeterol & 2 & 0 \\
Beclomethasone & 2 & 0 \\
Vancomycin & 1 & 0 \\
Formoterol fumarate & 1 & 0 \\
Amphotericin B & 0 & 1 \\
\hline
\end{tabular}

* All CF subjects were taking multiple medications 
Table 4 Micro-organism isolated from sputum of patients with cystic fibrosis"

\begin{tabular}{lll}
\hline Organism & $\begin{array}{l}\text { CF }- \text { Ps. aeruginosa colonised } \\
(\mathbf{n}=\mathbf{1 6})\end{array}$ & $\begin{array}{l}\text { CF - Ps. aeruginosa non-colonised } \\
(\mathbf{n}=\mathbf{1 3})\end{array}$ \\
\hline Pseudomonas aeruginosa & 16 & 0 \\
Staphylococcus aureus & 7 & 11 \\
Aspergillus fumigatus & 6 & 2 \\
Haemophilus influenzae & 0 & 8 \\
Oropharyngeal flora & 5 & 3 \\
Candida species & 1 & 2 \\
Burkholderia multivorans & 0 & 2 \\
Inquilinus limosus & 1 & 0 \\
Penicillium species & 0 & 1 \\
Stenotrophomonas maltophilia & 0 & 1 \\
Scedosporium apiospermum & 1 & 0 \\
Wangiella dermatitidis & 1 & 0
\end{tabular}

\# All CF subjects had multiple micro-organisms in the lung

biomarker for Ps. aeruginosa growth in the CF lung. We have confirmed that Ps. aeruginosa consistently produces 2-AA during in vitro growth on several types of media. Our analytical GC/MS technique also detected trace amounts of 2-AA in two of the three E. coli strains tested and in one of the three S. aureus strains, however, the level was two magnitudes lower than the 2-AA levels detected in Ps. aeruginosa cultures. Although Ps. aeruginosa produces millimol levels of 2-AA in ideal aerobic conditions, we have yet to determine whether 2 -AA production is dependent on the growth phase of the organisms or if it is affected by oxygen tension or nutrient supply. It is possible these conditions will differ between early lung colonisation and established Ps. aeruginosa infection, potentially affecting the performance of a breath test.

Our breath sampling system involves the collection of breath into a transportable device. Here, preliminary data showed that significant levels of background emissions from the Tedlar ${ }^{\bullet}$ bag itself (even after $\mathrm{N}_{2}$ flushing) do interfere with the 2-AA detection. Even in the deactivated glass sampling bulb 2-AA does not remain stable due to its reactive, polar chemical nature (11.73 hours (95\% CI 10.94, 12.52)). 2-Aminoacetophenone is thought to undergo oxidation to 2-nitroacetophenone but can be analysed accurately provided breath samples are processed promptly. A five parameter logistic function was fitted to the degradation data (Figure 5) and used to estimate $\mathrm{EC}_{50}$. Such models do not assume symmetry and it has been shown that any function with less than five parameters is unlikely to have the flexibility necessary to produce a high-quality fit to asymmetric sigmoidal dose-response data [22].

The analysis of specific markers for infectious diseases in breath is difficult due to the very low levels of volatiles present in the breath matrix [23,24]. However, GC/MS ${ }^{3}$ is a very sensitive technique and coupled with SPME preconcentration extremely low levels of analytes can be detected. Comparing spiked samples (ambient air spiked with the 2-AA reference standard) with real breath samples can be used for an assessment of a detection range provided the same experimental procedure is followed.

The most important finding of this study was that 2-AA was present above the limit of detection in a

Table 5 Survey of food consumption prior to 12 hours of breath collection

\begin{tabular}{llll}
\hline Food & Healthy controls $(\mathbf{n}=\mathbf{1 7})$ & CF - Ps. aeruginosa colonised $(\mathbf{n}=\mathbf{1 6})$ & CF - Ps. aeruginosa non-colonised $(\mathbf{n}=\mathbf{1 3})$ \\
\hline Milk & 10 & 7 & 9 \\
Cheese & 1 & 2 & 0 \\
Energy Drink & 1 & 2 & 2 \\
Coffee & 6 & 3 & 1 \\
Yoghurt & 2 & 4 & 1 \\
Green tea & 0 & 1 & 0 \\
Beer & 0 & 0 & 0 \\
Sweet corn & 0 & 0 & 0 \\
Corn chips & 0 & 1 & 1 \\
Honey & 0 & 2 & 0
\end{tabular}


higher proportion in the breath of subjects with CF who were colonised with Ps. aeruginosa, than both control groups (Figure 6). The peak integration values of 2-AA can be regarded as semi-quantitative only, but are consistent with the results of the analysis for presence or absence of 2-AA in breath, and support the conclusion that the likely source of the 2-AA are Ps. aeruginosa colonising the lungs. In contrast there was no evidence that $S$. aureus colonisation of the lung produced a positive 2-AA breath test despite trace amounts being detectable in the headspace of in vitro cultures. This preliminary study supports the concept that 2-AA may potentially serve as a biomarker of pseudomonas infection or colonisation in CF patients. This is consistent with previous studies of Aspergillus colonisation and Mycobacterium tuberculosis infection showing that specific biomarkers of these organisms are detectable in the breath by GC/MS techniques [24-26]. 2-Aminoacetophenone was also detectable in a small proportion of both healthy controls and subjects with CF who did not have evidence of Ps. aeruginosa on sputum sampling. The levels of 2-AA were very low and the origins of this signal are, at present, unknown.

As with any breath test, food, environment and medication are potential sources of the diagnostic marker. 2-Aminoacetophenone has been reported in foods and beverages namely corn, dairy, honey products and wine [27-29]. Breath testing immediately after the consumption of any one of these products may potentially give a false positive result. Investigation into whether ingested 2-AA rich food and beverages may increase 2-AA levels in the breath is currently ongoing. While ingested 2-AA may reach the breath it is possible that some or all may be metabolised by the host. It is not known whether

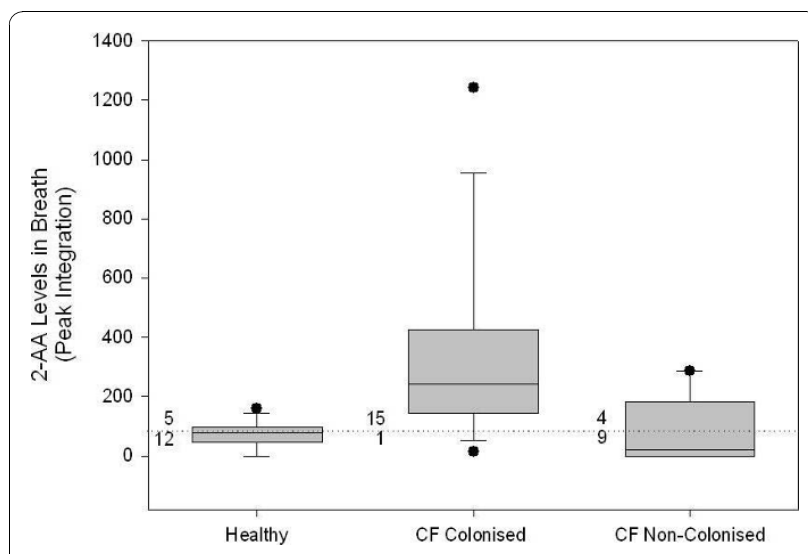

Figure 6 2-AA detection in breath. The peak integration values for 2-AA were significantly higher in CF patients colonised with Ps. aeruginosa than in CF patients not colonised $(p<0.001)$ with Ps. aeruginosa and healthy subjects $(p<0.0002)$. The numbers of subjects with detectable 2-AA in each group are shown. The lower limit of detection (peak integration of 88) is shown as a dotted line. prescribed or over the counter medication may also artificially increase or decrease the levels of 2-AA found in the breath but we have tested clinical preparations of tobramycin and colistin to exclude these as a potentially confounding source of 2-AA in this study. It is possible that some medications may breakdown to 2-AA which will require detailed investigations given the wide variety of medication taken by CF sufferers. A further possible source of 2-AA, particularly in CF patients is microbial colonisation of the mouth which was not detected on the sputum sampling. This may come from oral flora as well as Ps. aeruginosa. Further studies are needed to exclude these possible sources and it may be possible to reduce 2 -AA levels in healthy individuals by careful control of the experimental conditions.

One of the major problems with breath testing in general is the sampling technique. Only a small number of studies have been performed to investigate the factors that affect the concentration of endogenous VOC's on the breath before it exits the mouth [30]. While deactivated glass bulbs have been used successfully in previous studies [24-26] the most appropriate breath sampling technique for microbial derived VOCs has not yet been determined.

\section{Conclusions}

In conclusion our investigation supports the concept that 2-AA is a potential breath biomarker for Ps. aeruginosa and warrants further investigation as an indicator of $P$. aeruginosa colonisation or infection in the CF lung.

\section{Acknowledgements}

This work was funded by a New Zealand Lotteries Health Grant. The authors wish to thank the Microbiology Unit of the Canterbury Health Laboratories in Christchurch, especially Roslyn Podmore for her assistance. We would also like to thank Robyn Beach in the Respiratory Ward and Vivienne Isles \& Jaqueline Koortse in the Paediatrics Ward of Christchurch Public Hospital in Christchurch, New Zealand.

\section{Author details \\ ${ }^{1}$ University of Otago, Christchurch School of Medicine and Health Sciences, Department of Pathology, P.O. Box 4345, Christchurch, 8140, New Zealand. ${ }^{2}$ Leipzig University, Department for Inner Medicine, Neurology and Dermatology, Johannisallee 32, 04103 Leipzig, Germany. ${ }^{3}$ Canterbury District Health Board, Private Bag 4710, Christchurch, 8140 New Zealand.}

\section{Authors' contributions}

AST completed the experiments testing the stability of 2-AA in the current breath collection system. AST collected and analysed environmental air samples and breath samples, participated in the design of the study and drafted the manuscript. MS set up the GC/MS protocols for the detection of 2-AA and also completed the detection of 2-AA in bacterial cultures in vitro. PP participated in recruiting paediatric CF patients for breath analysis and in the final layout of the manuscript. ME was involved in study design and analysis of the result. ME also helped further improve the final manuscript draft. RL participated in recruiting adult CF patients from his clinics for breath analysis and the final layout of the manuscript. JP was integral in not only the study of the designs but also for the statistical analysis of all the results presented in this manuscript. SC participated in the design of the study, collection and analysis of clinical data, co-ordination of Respiratory Practitioners and the writing of the manuscript. All authors read and approved the final manuscript. 


\section{Competing interests}

The authors declare that they have no competing interests.

Received: 19 April 2010 Accepted: 7 November 2010

Published: 7 November 2010

\section{References}

1. Mann S: Uber den Geruchsstoff von Pseudomonas aeruginosa. Archiv fur Mikrobiologie 1966, 54:184-190.

2. Cox C, Parker J: Use of 2-aminoacetophenone production in identification of Pseudomonas aeruginosa. Journal of Clinical Microbiology 1979, 9(4):479-484.

3. Mann S: Chinazolinderivate bei Pseudomonaden. Archiv fur Mikrobiologie 1967, 56:324-329.

4. Labows J, McGinley K, Webster G, Leyden J: Headspace analysis of volatile metabolites of Pseudomonas aeruginosa and related species by gas chromatography-mass spectrometry. Journal of Clinical Microbiology 1980, 12(4):521-526.

5. Scotter J, Allardyce R, Langford V, Hill A, Murdoch D: The rapid evaluation of bacterial growth in blood cultures by selected ion flow tube-mass spectrometry (SIFT-MS) and comparison with the BacT/ALERT automated blood culture system. Journal of Microbiological Methods 2006, 65:628-631

6. Treggiari M, Rosenfeld M, Retsch-Bogart G, Gibson R, Ramsey B: Approach to eradication of initial Pseudomonas aeruginosa infection in children with cystic fibrosis. Pediatric Pulmonology 2007, 42:751-756

7. Reynolds H, Levine A, Wood R, Zierdt C, Dale D, Pennington J: Pseudomonas infections: persisting problems and current research to find new therapies. Annals of Internal Medicine 1975, 82:819-832.

8. Palmer K, Aye L, Whiteley M: Nutritional cues control Pseudomonas aeruginosa multicellular behaviour in cystic fibrosis sputum. Journal of Bacteriology 2007, 189(22):8079-2027.

9. Nixon G, Armstrong D, Carzino R, Carlin J, Olinsky A, Roberston C, Grimwood K: Clinical outcome after early Pseudomonas aeruginosa infection in cystic fibrosis. Journal of Pediatrics 2001, 138(5):699-704.

10. Dakin C, Numa A, Wang H, Morton J, Vertzyas C, Henry R: Inflammation, infection and pulmonary function in infants and young children with cystic fibrosis. American Journal of Respiratory and Critical Care Medicine 2002, 165(7):904-910

11. Hilliard T, Sukhani S, Francis J, Madden N, Rosenthal M, Balfour-Lynn I, Bush A, Davies J: Bronchoscopy following diagnosis with cystic fibrosis. Archives of Disease in Childhood 2006, 92(10):898-899.

12. Douglas T, Brennan S, Gar S, Berry L, Gangell C, Stick S, Clements B, Sly P: Acquisition and eradication of $P$. aeruginosa in young children with cystic fibrosis. European Respiratory Journal 2009, 33(2):305-311.

13. Rosenfeld M, Emerson J, Accurso F, Armstrong D, Castile R, Grimwood K, Hiatt P, McCoy K, McNamara S, Ramsey B, et al: Diagnostic accuracy of oropharyngeal cultures in infants and young children with cystic fibrosis. Pediatric Pulmonology 1999, 28:321-328.

14. Ramsey B, Wentz K, Smith A, Richardson M, Williams-Warren J, Hedges D, Gibson R, Redding G, Lent K, Harris K: Predictive value of oropharyngeal cultures for identifying lower airway bacteria in cystic fibrosis patients. American Review of Respiratory Disease 1991, 144(2):331-337.

15. Forbes B, Sahm D, Weissfeld A: Diagnostic Microbiology. Missouri: Mosby; Eleventh 2002

16. Mahenthiralingam E, Bishof J, Byrne S, Radomski C, Davies J, Av-Gay Y, Vandamme P: DNA-based diagnostic approaches for identification of Burkholderia cepacia complex, Burkholderia vietnamiensis, Burkholderia multivorans, Burkholderia stabilis, and Burkholderia cepacia genomovars I and III. Journal of Clinical Microbiology 2000, 38(9):3165-3173.

17. Atlas RM: Handbook of microbiological media. Kentucky: CRC Press; Third 2004.

18. Ritz C, Streiburg J: Bioassay analysis using R.J. Statist. Software 2010, 12(5).

19. $\mathrm{R}: \mathrm{A}$ language and environment for statistical computing. In $R$ Foundation for Statistical Computing. Edited by: Team RDC. Vienna, Austria; 2010.

20. Amann A, Smith D: Breath analysis for clinical diagnosis and therapeutic monitoring. Singapore: World Scientific Printers (S) Pte Ltd i First 2005.

21. Habs H, Mann S: Die bildung von ortho-aminoacetophenon durch apyacyanogene stamme von Pseudomonas aeruginosa. Zentralbl Bakteriol Parasitenkd Infektionskr hyg abt 1967, 203:473-477.
22. Gottschalk P, CDunn J: The five-parameter logistic: a characterization and comparison with the four-paramter logistic. Analytical biochemistry 2005, 343:54-65.

23. Syhre M, Scotter J, Chambers S: Investigation into the production of 2Pentylfuran by Aspergillus fumigatus and other respiratory pathogens in vitro and human breath samples. Medical Mycology 2007, 46:209-215.

24. Syhre M, Chambers S: The scent of Mycobacterium tuberculosis. Turberculosis 2008, 88(4):317-323.

25. Syhre M, Manning L, Harino P, Chambers S: The scent of Mycobacterium tuberculosis - part II. Tuberculosis 2009, 89:263-266.

26. Chambers S, Syhre M, Murdoch D, McCartin F, Epton M: Detection of 2pentylfuran in the breath of patients with Aspergillus fumigatus. Medical Mycology 2009, 47(5):468-476.

27. Schmarr H, Gan S, Sang W, Potouridis T: Analysis of 2-aminoacetophenone in wine using a stable isotope dilution assay and multidimensional gas chromatography-mass spectrometry. Journal of Chromatography A 2007, 1150:78-84

28. Fan W, Tsai I, Qian M: Analysis of 2-aminoacetophenone by directimmersion solid-phase microextraction and gas chromatography-mass spectrometry and its sensory impact in Chardonnay and Pinot gris wines. Food Chemistry 2007, 105:1144-1150.

29. Karagul-Yuceer $Y$, Cadwallader K, Drake M: Volatile flavor components of stored nonfat dry milk. Journal of Agricultural Food and Chemistry 2002, 50:305-312.

30. O'Hara M, O'Hehir S, Green S, Mayhew C: Development of a protocol to measure volatile organic compounds in human breath: a comparison of rebreathing and on-line single exhalations using proton transfer reaction mass spectrometry. Physiological Measurement 2008, 29:309-330.

\section{Pre-publication history}

The pre-publication history for this paper can be accessed here: http://www.biomedcentral.com/1471-2466/10/56/prepub

\section{doi:10.1186/1471-2466-10-56}

Cite this article as: Scott-Thomas et al.: 2-Aminoacetophenone as a potential breath biomarker for Pseudomonas aeruginosa in the cystic fibrosis lung. BMC Pulmonary Medicine 2010 10:56.

\section{Submit your next manuscript to BioMed Central and take full advantage of:}

- Convenient online submission

- Thorough peer review

- No space constraints or color figure charges

- Immediate publication on acceptance

- Inclusion in PubMed, CAS, Scopus and Google Scholar

- Research which is freely available for redistribution
C Biomed Central 